\title{
A Contact-Stabilized Newmark Method for Dynamical Contact Problems ${ }^{2,3}$
}

\footnotetext{
${ }^{1}$ Institute for Numerical Simulation, University of Bonn, Wegelerstraße 6, D-53115 Bonn

${ }^{2}$ Supported by the DFG Research Center MAtheon, "Mathematics for key technologies. Modelling, simulation, and optimization of real-world processes", Berlin

${ }^{3}$ Supported by the DFG, Collaborative Research Center (SFB) 611, "Singular Phenomena and Scaling in Mathematical Models", Bonn
} 



\title{
A Contact-Stabilized Newmark Method for Dynamical Contact Problems
}

\author{
Peter Deufhard, Rolf Krause, Susanne Ertel
}

\begin{abstract}
The numerical integration of dynamical contact problems often leads to instabilities at contact boundaries caused by the non-penetration condition between bodies in contact. Even a recent energy dissipative modification due to Kane et al. (1999), which discretizes the non-penetration constraints implicitly, is not able to circumvent artificial oscillations. For this reason, the present paper suggests a contact stabilization which avoids artificial oscillations at contact interfaces and is also energy dissipative. The key idea of this contact stabilization is an additional $L^{2}$-projection at contact interfaces, which can easily be added to any existing time integration scheme. In case of a lumped mass matrix, this projection can be carried out completely locally, thus creating only negligible additional numerical cost. For the new scheme, an elementary analysis is given, which is confirmed by numerical findings in an illustrative test example (Hertzian two body contact).
\end{abstract}




\section{Contents}

$\begin{array}{ll}\text { Introduction } & 1\end{array}$

1 Dynamical contact problems 2

1.1 Strong and variational problem formulation . . . . . . . 2

1.2 Conservation properties . . . . . . . . . . . . 6

2 Newmark schemes $\quad 8$

2.1 Classical scheme . . . . . . . . . . . . . . . . . . . . 9

2.2 A recent dissipative modification . . . . . . . . . . . . 10

2.3 Contact stabilization . . . . . . . . . . . . . . 13

3 A two body Hertzian contact problem 15

$\begin{array}{ll}\text { References } & 18\end{array}$ 


\section{Introduction}

Dynamical contact problems play an important role in mechanics and biomechanics. One of the main difficulties for the development of efficient and reliable discretization schemes in time and space is the non-penetration condition at the contact interfaces between bodies. Their improper handling might lead to spurious numerical oscillations at the interfaces, therefore spoiling the accuracy of the obtained solution and slowing down the overall computing time considerably. For the design of time integration schemes for contact problems, various approaches can be found in the literature, see [12] for a monographic survey. One of the most popular time discretization schemes for dynamical contact problems is the Newmark method. In the absence of contact, the classical Newmark scheme is well-known to be symplectic $[5,14]$ and of second order consistency in the symmetric case. For contact problems, Kane et. al [6] recently suggested a stabilized modification, where the contact forces are treated fully implicitly. In contrast to the classical scheme, their modified scheme is energy dissipative. Unfortunately, artificial oscillations may show up.

The present paper deals with a further modification, which in the presence of contact is also energy dissipative, but avoids the artificial oscillations. Our stabilization is motivated by the requirement that at contact interfaces the contact forces should balance. This leads us to an additional discrete $L^{2}$-projection in each time step which can easily be incorporated into the Newmark scheme by simply changing the predictor step. The reason for this projection to be discrete can be found in the fact that by discretization in space the boundary gets assigned to a mass, although for the continuous case the boundary has measure zero. Through the Newmark scheme, this discrete boundary mass gets translated into forces at the contact boundary, which can be found to be one of the main causes for the artificial oscillations at the interface. Now, by means of our contact-stabilization, the non-physical part of these boundary forces is removed. We want to mention that recently a different approach for avoiding the oscillations at the boundary has been presented which is based on completely removing the mass in a small strip on the boundary, see [7]. Our approach, however, leaves the mass matrix unchanged and can easily be applied for arbitrary spatial discretization.

The paper is organized as follows. In Section 1, we first present basic features of the continuous dynamical contact problem. Both strong and weak problem formulation as well as conservation properties are discussed. In Sec- 
tion 2, we synoptically present the classical Newmark scheme, its modification by Kane et al., and our new contact-stabilized scheme. Finally, in Section 3, we illustrate our elementary theoretical analysis by numerical findings at a Hertzian contact problem. Throughout the paper, unless explicitly specified otherwise, we ignore the spatial discretization, which has been described in detail in $[9,10,15]$.

\section{Dynamical contact problems}

Before we dive into the details of numerical simulation, we want to study the main properties of the continuous dynamical contact problem. In Section 1.1 below, we present the strong and the weak problem formulation in parallel. After that, in Section 1.2, we discuss conservation properties, which will play a major role in the numerical realization as worked out in the subsequent Section 2.

\subsection{Strong and variational problem formulation}

In what follows, we give the strong and the weak problem formulations for the dynamic contact between two elastic bodies. The weak formulation is given in terms of a variational inequality.

Notation. All domains treated here are understood to be subsets in $\mathbb{R}^{d}$ with $d=2,3$ and corresponding running indices $1 \leq i, j, l, m \leq d$ throughout the paper. Let the two bodies be identified with the domains $\Omega^{K}, K \in$ $\{S, M\}$ where $S$ and $M$ stand for slave and master body, respectively. Let the solution be decomposed according to $\mathbf{u}=\left(\mathbf{u}^{S}, \mathbf{u}^{M}\right)$. Each of the boundaries $\partial \Omega^{K}$ with associated outward directed normal $\boldsymbol{\nu}^{K}$ is decomposed into three disjoint parts: $\Gamma_{D}^{K}$, the Dirichlet boundary, $\Gamma_{N}^{K}$, the Neumann boundary, and $\Gamma_{C}^{K}$, the possible contact boundary, see Figure 1. The actual contact boundary is not known in advance, but is assumed to be contained in a compact strict subset of $\Gamma_{C}^{K}$. Set $\Omega=\Omega^{S} \cup \Omega^{M}$ and $\Gamma_{*}=\Gamma_{*}^{S} \cup \Gamma_{*}^{M}$ for $* \in\{D, N, C\}$.

Tensor and vector quantities are written in bold characters, e.g., $\boldsymbol{\sigma}$ and $\mathbf{v}$ with components $\sigma_{i j}$ and $v_{i}$. The partial derivative with respect to the spatial variable $x_{j}$ is indicated by a subindex $j$, e.g., $\mathbf{v}_{, j}$. The summation convention is enforced on all repeated indices ranging in $1, \ldots, d$. Let $\mathbf{L}^{2}\left(\Omega^{K}\right)=$ 


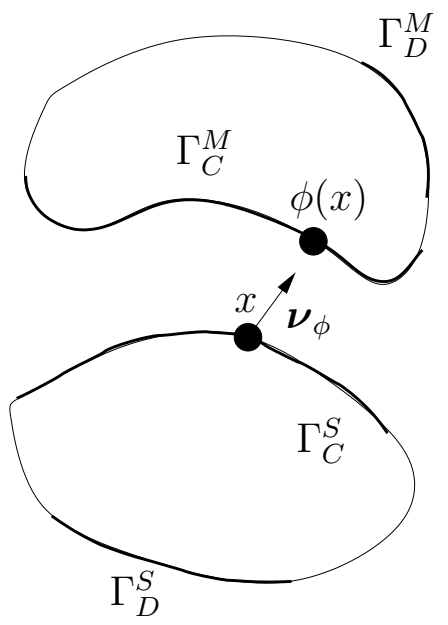

Figure 1: Two body contact problem and decomposition into $\Gamma_{D}, \Gamma_{N}$ and $\Gamma_{C}$

$\left(L^{2}\left(\Omega^{K}\right)\right)^{d}$ and $\mathbf{L}^{2}=\mathbf{L}^{2}\left(\Omega^{S}\right) \times \mathbf{L}^{2}\left(\Omega^{M}\right)$. The Sobolev spaces of displacements with weak derivative in $\mathbf{L}^{2}\left(\Omega^{K}\right)$ are denoted as $\mathbf{H}^{1}\left(\Omega^{K}\right)=\left(H^{1}\left(\Omega^{K}\right)\right)^{d}$. For Dirichlet boundary conditions we define the subspaces $\mathbf{H}_{D}^{1}\left(\Omega^{K}\right)=\{\mathbf{v} \mid \mathbf{v} \in$ $\left.\mathbf{H}^{1}\left(\Omega^{K}\right),\left.\mathbf{v}\right|_{\Gamma_{D}^{K}}=\mathbf{0}\right\}$, and $\mathbf{H}_{D}^{1}=\mathbf{H}_{D}^{1}\left(\Omega^{S}\right) \times \mathbf{H}_{D}^{1}\left(\Omega^{M}\right)$. Let $\mathbf{H}_{D}^{1}$ be equipped with the norm

$$
\|\mathbf{u}\|_{\mathbf{H}_{D}^{1}}^{2}=\left\|\mathbf{u}^{S}\right\|_{\mathbf{H}^{1}\left(\Omega^{S}\right)}^{2}+\left\|\mathbf{u}^{M}\right\|_{\mathbf{H}^{1}\left(\Omega^{M}\right)}^{2} .
$$

Scalar products are written in the form $(\cdot, \cdot)_{\mathbf{L}^{2}\left(\Omega^{K}\right)}$ and $(\cdot, \cdot)_{\mathbf{H}^{1}\left(\Omega^{K}\right)}$ with induced norms $\|\mathbf{v}\|_{\mathbf{L}^{2}\left(\Omega^{K}\right)}^{2}=(\mathbf{v}, \mathbf{v})_{\mathbf{L}^{2}\left(\Omega^{K}\right)},\|\mathbf{v}\|_{\mathbf{H}^{1}\left(\Omega^{K}\right)}^{2}=(\mathbf{v}, \mathbf{v})_{\mathbf{H}^{1}\left(\Omega^{K}\right)}$.

Elasticity model. The materials under consideration are assumed to be linearly elastic, i.e. the stresses $\boldsymbol{\sigma}$ satisfy Hooke's law

$$
\sigma_{i j}\left(\mathbf{u}^{K}\right)=E_{i j m l}^{K} u_{l, m}^{K},
$$

where Hooke's tensor $\mathbf{E}^{\mathbf{K}}=\left(E_{i j m l}^{K}\right)$ is sufficiently smooth (with $E_{i j m l}^{K} \in$ $L^{\infty}\left(\Omega^{K}\right)$ ), symmetric, and uniformly positive definite. The linearized strain tensor is

$$
\boldsymbol{\varepsilon}\left(\mathbf{u}^{K}\right)=\frac{1}{2}\left(\nabla \mathbf{u}^{K}+\left(\nabla \mathbf{u}^{K}\right)^{T}\right)
$$

Unfortunately, this means that our scheme is not invariant under rigid body motions. 
Non-penetration condition. At the contact interface $\Gamma_{C}$, the two bodies may come into contact but must not penetrate each other. In order to derive a non-penetration condition, we assume a bijective mapping $\phi: \Gamma_{C}^{S} \longrightarrow \Gamma_{C}^{M}$ between the two possible contact surfaces to be given. Following [3], we define linearized non-penetration with reference to $\phi$ by

$$
[\mathbf{u} \cdot \boldsymbol{\nu}]_{\phi}(x, t)=\mathbf{u}^{S}(x, t) \cdot \boldsymbol{\nu}_{\phi}(x)-\mathbf{u}^{M}(\phi(x), t) \cdot \boldsymbol{\nu}_{\phi}(x) \leq g(x), \quad x \in \Gamma_{C}^{S} .
$$

This condition is given with respect to the initial gap

$$
\Gamma_{C}^{S} \ni x \mapsto g(x)=|x-\phi(x)| \in \mathbb{R}
$$

between the two bodies in the reference configuration and we have set

$$
\boldsymbol{\nu}_{\phi}=\left\{\begin{array}{cl}
\frac{\phi(x)-x}{|\phi(x)-x|}, & \text { if } \quad x \neq \phi(x), \\
\boldsymbol{\nu}^{S}(x)=-\boldsymbol{\nu}^{M}(x), & \text { if } \quad x=\phi(x)
\end{array}\right.
$$

where $|\cdot|$ stands for the Euclidean vector norm in $\mathbb{R}^{d}$.

Under certain assumptions on $\phi$ and on the geometry of the deformed configuration (see [3]), the above defined non-penetration condition (1.1) is a close approximation of the geometrical non-penetration condition. In this case we call $[\mathbf{v} \cdot \boldsymbol{\nu}]_{\phi}$ the jump of $\mathbf{v}$ in normal direction at the interface. We define the contact traction $\hat{\boldsymbol{\sigma}}\left(\mathbf{u}^{K}\right)=\boldsymbol{\sigma}(\mathbf{u}) \boldsymbol{\nu}^{K}$. The equilibrium of the contact forces at the contact boundary is defined by

$$
\int_{\Gamma_{C}^{S}} \hat{\boldsymbol{\sigma}}\left(\mathbf{u}^{S}\left(x^{S}, t\right)\right) \cdot \mathbf{v}^{M}\left(\phi\left(x^{S}\right)\right) d a^{S}=-\int_{\Gamma_{C}^{M}} \hat{\boldsymbol{\sigma}}\left(\mathbf{u}^{M}\left(x^{M}, t\right)\right) \cdot \mathbf{v}^{M}\left(x^{M}\right) d a^{M}
$$

for every function $\mathbf{v}^{M} \in \mathbf{H}^{1 / 2}\left(\Gamma_{C}^{M}\right)$. Introducing the transfer operator $\Phi$ : $\mathbf{L}^{2}\left(\Gamma_{C}^{M}\right) \longrightarrow \mathbf{L}^{2}\left(\Gamma_{C}^{S}\right)$ and its adjoint $\Phi^{*}$, we can rewrite (1.2) as

$$
\hat{\boldsymbol{\sigma}}\left(\mathbf{u}^{M}\right)=-\Phi^{*} \hat{\boldsymbol{\sigma}}\left(\mathbf{u}^{S}\right)
$$

on $\Gamma_{C}^{M}$, see again [3]. We therefore can define the normal and tangential contact stresses by $\sigma_{\nu_{\phi}}$ and $\boldsymbol{\sigma}_{t}=\hat{\boldsymbol{\sigma}}-\sigma_{\nu_{\phi}} \cdot \boldsymbol{\nu}_{\phi}$. 
Strong formulation. This formulation consists of the equilibrium conditions (1.4a) in $\Omega^{S}$ and $\Omega^{M}$, the boundary conditions (1.4b), and (1.4c) on $\Gamma_{D}$ and $\Gamma_{N}$ and the contact conditions $(1.4 \mathrm{~d})-(1.4 \mathrm{~h})$. Thus we arrive at

$$
\begin{aligned}
\rho \ddot{u}_{i}-\sigma_{i j}(\mathbf{u})_{, j} & =f_{i} & & \text { in } \Omega \times[0, T] \\
\mathbf{u} & =0 & & \text { on } \Gamma_{D} \times[0, T] \\
\sigma_{i j}(\mathbf{u}) \cdot \nu_{j} & =\pi_{i} & & \text { on } \Gamma_{N} \times[0, T] \\
\hat{\boldsymbol{\sigma}}\left(\mathbf{u}^{M}\right) & =-\Phi^{*} \hat{\boldsymbol{\sigma}}\left(\mathbf{u}^{S}\right) & & \text { on } \Gamma_{C} \times[0, T] \\
\sigma_{\nu_{\phi}} & \leq 0 & & \text { on } \Gamma_{C} \times[0, T] \\
{[\mathbf{u} \cdot \boldsymbol{\nu}]_{\phi} } & \leq g & & \text { on } \Gamma_{C} \times[0, T] \\
\left([\mathbf{u} \cdot \boldsymbol{\nu}]_{\phi}-g\right) \sigma_{\nu_{\phi}} & =0 & & \text { on } \Gamma_{C} \times[0, T] \\
\boldsymbol{\sigma}_{t}(\mathbf{u}) & =0 & & \text { on } \Gamma_{C} \times[0, T] \\
\mathbf{u}(x, 0)=\mathbf{u}_{0}(x), \dot{\mathbf{u}}(x, 0) & =\dot{\mathbf{u}}_{0}(x) & & \text { in } \Omega
\end{aligned}
$$

Due to the boundary condition (1.4e), only compressive normal stresses at the contact interface are allowed; by the complementarity condition $(1.4 \mathrm{~g})$ the normal stresses must vanish in the absence of contact.

Variational formulation. For the weak formulation, let the convex set of all admissible displacements be denoted by

$$
\mathcal{K}=\left\{\mathbf{v} \in \mathbf{H}_{D}^{1} \mid[\mathbf{v} \cdot \boldsymbol{\nu}]_{\phi} \leq g\right\}
$$

and the bilinear form $a(\cdot, \cdot)$ by

$$
a(\mathbf{v}, \mathbf{w})=\sum_{K \in\{S, M\}} \int_{\Omega^{K}} \boldsymbol{\sigma}\left(\mathbf{v}^{K}\right): \boldsymbol{\varepsilon}\left(\mathbf{w}^{K}\right) d x=f_{\mathrm{int}}(\mathbf{v}) \mathbf{w}, \quad \mathbf{v}, \mathbf{w} \in \mathbf{H}_{D}^{1}
$$

For the data assume that $\mathbf{f}(\cdot, t) \in \mathbf{L}^{2}, \boldsymbol{\pi}(\cdot, t) \in \mathbf{H}^{-1 / 2}\left(\Gamma_{N}\right)$ for all $t \in[0, T]$. On $\mathbf{H}_{D}^{1}$, the linear functional $f_{\text {ext }}$, which accounts for the volume forces and the tractions on the Neumann boundary, is given by

$$
f_{\mathrm{ext}}(\mathbf{v})=(\mathbf{f}, \mathbf{v})_{\mathbf{L}^{2}}+(\boldsymbol{\pi}, \mathbf{v})_{\mathbf{L}^{2}\left(\Gamma_{N}\right)} .
$$

In what follows, the $\mathbf{L}^{2}$ scalar-product is understood to be weighted by the density $\rho(x)$. Hence we may set $\rho=1$ for ease of presentation. 
We are now ready to write our problem in weak formulation as the hyperbolic variational inequality: For every $t \in[0, T]$ find $\mathbf{u}(\cdot, t) \in \mathcal{K}$ with $\ddot{\mathbf{u}}(\cdot, t) \in \mathbf{L}^{2}$ such that

$$
(\ddot{\mathbf{u}}, \mathbf{v}-\mathbf{u})_{\mathbf{L}^{2}}+a(\mathbf{u}, \mathbf{v}-\mathbf{u}) \geq f_{\text {ext }}(\mathbf{v}-\mathbf{u}), \quad \mathbf{v} \in \mathcal{K} .
$$

This formulation is derived via integrating by parts and exploiting the boundary conditions, see [2] and [8].

A formulation equivalent to (1.6) is derived incorporating the constraints $\mathbf{v}(t) \in \mathcal{K}$ for almost all $t \in[0, T]$ by the characteristic functional $I_{\mathcal{K}}(\mathbf{v})$,

$$
I_{\mathcal{K}}(\mathbf{v})=\left\{\begin{array}{cc}
0, & \text { if } \mathbf{v} \in \mathcal{K} \\
\infty, & \text { else }
\end{array}, \quad \mathbf{v} \in \mathbf{H}_{D}^{1} .\right.
$$

Upon representing the internal and external forces according to

$$
(\mathbf{F}(\mathbf{w}), \mathbf{v})_{\mathbf{L}^{2}}=a(\mathbf{w}, \mathbf{v})-f_{\text {ext }}(\mathbf{v}), \quad \mathbf{w}, \mathbf{v} \in \mathbf{H}_{D}^{1},
$$

the variational inequality (1.6) can be reformulated as the variational inclusion

$$
0 \in \ddot{\mathbf{u}}+\mathbf{F}(\mathbf{u})+\partial I_{\mathcal{K}}(\mathbf{u})
$$

utilizing the subdifferential $\partial I_{\mathcal{K}}$ of $I_{\mathcal{K}}$ (see, e.g., [4]). Once this variational inequality has been solved, we define the contact forces $\mathbf{F}_{\text {con }}$ as

$$
\begin{aligned}
\left(\mathbf{F}_{\mathrm{con}}(\mathbf{u}), \mathbf{v}\right)_{\mathbf{L}^{2}} & =(\ddot{\mathbf{u}}+\mathbf{F}(\mathbf{u}), \mathbf{v})_{\mathbf{L}^{2}} \\
& =(\ddot{\mathbf{u}}, \mathbf{v})_{\mathbf{L}^{2}}+a(\mathbf{u}, \mathbf{v})-f_{\text {ext }}(\mathbf{v}),
\end{aligned}
$$

where we have used (1.7) in the second line. Upon exploiting (1.6), for $\mathbf{v} \in \mathcal{K}$, we arrive at

$$
\begin{aligned}
\left(\mathbf{F}_{\text {con }}(\mathbf{u}), \mathbf{v}-\mathbf{u}\right)_{\mathbf{L}^{2}} & =(\ddot{\mathbf{u}}, \mathbf{v}-\mathbf{u})_{\mathbf{L}^{2}}+a(\mathbf{u}, \mathbf{v}-\mathbf{u})-f_{\operatorname{ext}}(\mathbf{v}-\mathbf{u}) \\
& \geq 0 .
\end{aligned}
$$

Summarizing, we may write Newton's equation for the constrained case as

$$
\ddot{\mathbf{u}}=-\mathbf{F}(\mathbf{u})+\mathbf{F}_{\text {con }} .
$$

\subsection{Conservation properties}

Since we cannot expect the energy to be constant in time in case of nonvanishing external forces acting on the volume or stemming from Dirichlet boundaries, we examine conservation under the necessary assumptions $\Gamma_{D}=$ $\emptyset$ and $f_{\text {ext }}=0$. Under this assumption we discuss the following conservation properties (see, e.g., [13]): 
Linear momentum $\mathcal{L}$. Inserting the constant trial function $\mathbf{w} \in \mathbb{R}^{d}$ into the variational formulation (1.6), one can easily check

$$
\frac{\mathrm{d}}{\mathrm{d} t} \mathcal{L}=\frac{\mathrm{d}}{\mathrm{d} t}\left(\int_{\Omega} \dot{\mathbf{u}} d x\right)=0
$$

Angular momentum $\mathcal{J}$. In order to show conservation of angular momentum, we would have to verify that

$$
\frac{\mathrm{d}}{\mathrm{d} t} \mathcal{J}=\frac{\mathrm{d}}{\mathrm{d} t}\left(\int_{\Omega} \mathbf{u} \times \dot{\mathbf{u}} d x\right)=0
$$

In [13], Laursen and Chawla inserted a trial function $\mathbf{w} \times \mathbf{u}$ with $\mathbf{w}$ constant and introduced a solution dependent surface-to-surface mapping $\tilde{\Phi}(\mathbf{u}, \cdot)$. Upon integrating by parts, they arrived at the boundary integral

$$
\int_{\Gamma_{C}^{S}} \mathbf{w} \cdot\left[\hat{\boldsymbol{\sigma}} \times\left(\mathbf{u}^{S}-\mathbf{u}^{M} \circ \tilde{\Phi}\right)\right] d a^{S} .
$$

This integral vanishes due to the collinearity of the gap $\left(\mathbf{u}^{S}-\mathbf{u}^{M} \circ \tilde{\Phi}\right)$ and the contact traction $\hat{\boldsymbol{\sigma}}$. Unfortunately, in our case, the above collinearity cannot be expected to hold, since our transfer operator $\Phi$ originates from the linearized non-penetration condition $(1.1)$ and, hence, $\Phi \neq \tilde{\Phi}$. From the point of view of the spatial problems, the linearized non-penetration condition (1.1) is consistent with the overall modeling assumption of small deformations, where angular momenta are of less importance. Moreover, in combination with the linear material law, this approach gives rise to a convex constrained minimization problem for fixed time $t$, thereby preserving the convex structure of the unconstrained spatial problem.

Energy $\mathcal{E}$. In our context, the energy has the form

$$
\mathcal{E}(\mathbf{u})=\mathcal{E}_{\text {kin }}(\mathbf{u})+\frac{1}{2} a(\mathbf{u}, \mathbf{u}),
$$

where

$$
\mathcal{E}_{\text {kin }}(\mathbf{u})=\frac{1}{2} \int_{\Omega}|\dot{\mathbf{u}}|^{2} d x .
$$


The proof of energy conservation typically exploits an additional requirement at the contact boundary, known as persistency condition,

$$
\sigma_{\nu_{\phi}} \frac{\mathrm{d}}{\mathrm{d} t}\left([\mathbf{u} \cdot \boldsymbol{\nu}]_{\phi}-g\right)=0 \text { on } \Gamma_{C}
$$

Assuming $\dot{\mathbf{u}}$ to be sufficiently smooth we may use it as a trial function and reformulate (1.14) as which leads to

$$
\begin{aligned}
\frac{\mathrm{d}}{\mathrm{d} t} \mathcal{E} & =\frac{\mathrm{d}}{\mathrm{d} t}\left(\frac{1}{2} \int_{\Omega}|\dot{\mathbf{u}}|^{2} d x+\frac{1}{2} \int_{\Omega} \boldsymbol{\sigma}(\mathbf{u}): \boldsymbol{\varepsilon}(\mathbf{u}) d x\right) \\
& =\int_{\Gamma_{C}^{S}} \hat{\boldsymbol{\sigma}}\left(\mathbf{u}^{S}\right) \cdot\left(\dot{\mathbf{u}}^{S}\left(x^{S}\right)-\dot{\mathbf{u}}^{M}\left(\phi\left(x^{S}\right)\right) \mathrm{d} a^{S}\right. \\
& =0 .
\end{aligned}
$$

The above condition (1.14), sufficient for energy conservation, is widely disputed, since its physical meaning is unclear (see, e.g., [12]). For this reason it will not enter in the construction of the investigated algorithms.

As a consequence of the above considerations we will investigate the various schemes with respect to conservation of linear momentum and energy, see below.

\section{Newmark schemes}

This section deals with the Rothe discretization of dynamical contact problems, i.e., a discretization first in time and then in space. Details of the spatial discretization (adaptive finite elements) are omitted here and can be found in $[9,10]$. Unless explicitly specified otherwise, we do not distinguish between the continuous and the corresponding spatially discretized quantities in our notation. However, whenever particular properties of the spatial discretization are exploited, this will be indicated.

Discretization in time. Let $\tau>0$ denote a discrete timestep subdividing the considered time interval $[0, T]$ into equidistant parts $\left[t^{n}, t^{n+1}\right]$ with the discrete times $t^{n}=n \tau$ for all $n \in\{0, \ldots, T / \tau\}$. 


\subsection{Classical scheme}

We start by considering the time discretization of the differential equation (1.11) in the dual of $\mathbf{H}_{D}^{1}$. The classical Newmark scheme [12] is based on Taylor expansions of the displacements $\mathbf{u}$ and the velocities $\dot{\mathbf{u}}$. Neglecting terms of higher order and introducing the traditional parameters $\gamma, 2 \beta \in[0,1]$ leads to

$$
\begin{aligned}
& \mathbf{u}^{n+1}=\mathbf{u}^{n}+\tau \dot{\mathbf{u}}^{n}+\frac{\tau^{2}}{2}\left((1-2 \beta) \ddot{\mathbf{u}}^{n}+2 \beta \ddot{\mathbf{u}}^{n+1}\right), \\
& \dot{\mathbf{u}}^{n+1}=\dot{\mathbf{u}}^{n}+\tau\left((1-\gamma) \ddot{\mathbf{u}}^{n}+\gamma \ddot{\mathbf{u}}^{n+1}\right)
\end{aligned}
$$

with $\mathbf{u}^{n}$ approximating $\mathbf{u}\left(\cdot, t^{n}\right)$. For ease of writing we introduce the shorthand notation

$$
\mathbf{F}^{\lambda}\left(\mathbf{u}^{n}, \mathbf{u}^{n+1}\right)=(1-\lambda) \mathbf{F}\left(\mathbf{u}^{n}\right)+\lambda \mathbf{F}\left(\mathbf{u}^{n+1}\right), \quad \lambda \in[0,1] .
$$

Upon inserting (1.8), we obtain the time discretization of our contact problem (1.6) as

$$
\begin{aligned}
\mathbf{u}_{\text {pred }}^{n+1} & =\mathbf{u}^{n}+\tau \dot{\mathbf{u}}^{n} \\
0 & \in \mathbf{u}^{n+1}-\mathbf{u}_{\text {pred }}^{n+1}+\frac{1}{2} \tau^{2}\left(\mathbf{F}^{2 \beta}\left(\mathbf{u}^{n}, \mathbf{u}^{n+1}\right)-\widetilde{\mathbf{F}}_{\text {con }}^{2 \beta}\left(\mathbf{u}^{n}, \mathbf{u}^{n+1}\right)\right) \\
\dot{\mathbf{u}}^{n+1} & =\dot{\mathbf{u}}^{n}-\tau\left(\mathbf{F}^{\gamma}\left(\mathbf{u}^{n}, \mathbf{u}^{n+1}\right)-\mathbf{F}_{\text {con }}^{\gamma}\left(\mathbf{u}^{n}, \mathbf{u}^{n+1}\right)\right)
\end{aligned}
$$

where

$$
\widetilde{\mathbf{F}}_{\text {con }}^{2 \beta}\left(\mathbf{u}^{n}, \mathbf{u}^{n+1}\right)=(1-2 \beta) \mathbf{F}_{\text {con }}\left(\mathbf{u}^{n}\right)-2 \beta \partial I_{\mathcal{K}}\left(\mathbf{u}^{n+1}\right) .
$$

Note that $\widetilde{\mathbf{F}}_{\text {con }}^{2 \beta}\left(\mathbf{u}^{n}, \mathbf{u}^{n+1}\right) \neq \mathbf{F}_{\text {con }}^{2 \beta}\left(\mathbf{u}^{n}, \mathbf{u}^{n+1}\right)$ is needed in the second line, since $\mathbf{F}_{\text {con }}\left(\mathbf{u}^{n+1}\right)$ is not available before the variational inclusion has been solved.

For the unconstrained problem, setting $\gamma=\frac{1}{2}$ ensures second order consistency. For $2 \beta>0$, the variational inclusion in the second line of $(2.17)$ has to be solved in each time step. For $2 \beta=0$, the scheme is explicit and unstable. It can only be applied in a method of lines approach, i.e., after discretization in space first - observing a CFL-condition. For $2 \beta \geq \frac{1}{2}\left(\gamma+\frac{1}{2}\right)^{2}$ the algorithm is unconditionally stable. The usual choice of $2 \beta=\frac{1}{2}$ optimizes the damping of high frequencies that may originate from the spatial discretization. For this symmetric case, the Newmark scheme (2.17) reduces to the trapezoidal rule, which is energy conserving [5].

For our constrained problem (with $2 \beta=\gamma=\frac{1}{2}$ ), the total energy of the system at the end of a new timestep is conserved only, if the actual contact boundary has not changed during this timestep, see [12]. However, 
for the space-time-discrete scheme, the detection of a new contact point will decrease the energy of the system, the release of an existing contact point will increase it. Unfortunately, loss and regain of energy do not balance, so that the energy cannot be guaranteed to remain bounded during time integration. In particular, an undesirable energy blow-up may occur. That is why a dissipative modification has been proposed, which will be described next.

\subsection{A recent dissipative modification}

In [6], C. Kane et al. proposed a fully implicit treatment of the contact forces leading to the following variant of Newmark's scheme

$$
\begin{aligned}
\mathbf{u}_{\text {pred }}^{n+1} & =\mathbf{u}^{n}+\tau \dot{\mathbf{u}}^{n}, \\
0 & \in \mathbf{u}^{n+1}-\mathbf{u}_{\text {pred }}^{n+1}+\frac{1}{2} \tau^{2}\left(\mathbf{F}^{2 \beta}\left(\mathbf{u}^{n}, \mathbf{u}^{n+1}\right)+\partial I_{\mathcal{K}}\left(\mathbf{u}^{n+1}\right)\right) \\
\dot{\mathbf{u}}^{n+1} & =\dot{\mathbf{u}}^{n}-\tau\left(\mathbf{F}^{\gamma}\left(\mathbf{u}^{n}, \mathbf{u}^{n+1}\right)-\mathbf{F}_{\text {con }}\left(\mathbf{u}^{n+1}\right)\right) .
\end{aligned}
$$

Clearly, compared to the classical scheme, the term $\partial I_{\mathcal{K}}\left(\mathbf{u}^{n+1}\right)$ replaces the term $\widetilde{\mathbf{F}}_{\text {con }}^{2 \beta}\left(\mathbf{u}^{n}, \mathbf{u}^{n+1}\right)$, thus introducing an additional implicitness to the scheme.

Theorem 2.1 Consider Algorithm (2.18) for $\gamma=2 \beta=\frac{1}{2}$. If no contact occurs, this algorithm is energy conserving. If contact occurs, it can be energy dissipative.

Proof 2.1 Upon combining the second and the third line of Algorithm (2.18), we obtain

$$
\begin{aligned}
\dot{\mathbf{u}}^{n+1}-\dot{\mathbf{u}}^{n} & =\frac{2}{\tau}\left(\mathbf{u}^{n+1}-\mathbf{u}_{\text {pred }}^{n+1}\right), \\
\dot{\mathbf{u}}^{n+1}+\dot{\mathbf{u}}^{n} & =\frac{2}{\tau}\left(\mathbf{u}^{n+1}-\mathbf{u}^{n}\right) .
\end{aligned}
$$

Therefore the difference in kinetic energy evaluated at the end points of the 
time interval $\left[t^{n}, t^{n+1}\right]$ can be calculated as

$$
\begin{aligned}
\mathcal{E}_{\text {kin }}\left(\mathbf{u}^{n+1}\right)-\mathcal{E}_{\text {kin }}\left(\mathbf{u}^{n}\right)= & \frac{1}{2} \int_{\Omega}\left(\dot{\mathbf{u}}^{n+1}-\dot{\mathbf{u}}^{n}\right) \cdot\left(\dot{\mathbf{u}}^{n+1}+\dot{\mathbf{u}}^{n}\right) d x \\
= & \frac{2}{\tau^{2}} \int_{\Omega}\left(\mathbf{u}^{n+1}-\mathbf{u}_{\text {pred }}^{n+1}\right) \cdot\left(\mathbf{u}^{n+1}-\mathbf{u}^{n}\right) d x \\
= & -\int_{\Omega}\left(\mathbf{F}^{1 / 2}\left(\mathbf{u}^{n}, \mathbf{u}^{n+1}\right)-\mathbf{F}_{\text {con }}\left(\mathbf{u}^{n+1}\right)\right)\left(\mathbf{u}^{n+1}-\mathbf{u}^{n}\right) d x \\
= & -\frac{1}{2} a\left(\mathbf{u}^{n+1}+\mathbf{u}^{n}, \mathbf{u}^{n+1}-\mathbf{u}^{n}\right) \\
& +f_{\text {ext }}\left(\mathbf{u}^{n+1}-\mathbf{u}^{n}\right)+\int_{\Omega} \mathbf{F}_{\operatorname{con}}\left(\mathbf{u}^{n+1}\right)\left(\mathbf{u}^{n+1}-\mathbf{u}^{n}\right) d x .
\end{aligned}
$$

The change in total energy is

$$
\begin{aligned}
\mathcal{E}\left(\mathbf{u}^{n+1}\right)-\mathcal{E}\left(\mathbf{u}^{n}\right)= & \mathcal{E}_{\text {kin }}\left(\mathbf{u}^{n+1}\right)-\mathcal{E}_{\text {kin }}\left(\mathbf{u}^{n}\right) \\
& +\frac{1}{2} a\left(\mathbf{u}^{n+1}, \mathbf{u}^{n+1}\right)-f_{\text {ext }}\left(\mathbf{u}^{n+1}\right)-\frac{1}{2} a\left(\mathbf{u}^{n}, \mathbf{u}^{n}\right)+f_{\text {ext }}\left(\mathbf{u}^{n}\right) \\
= & \int_{\Omega} \mathbf{F}_{\mathrm{con}}\left(\mathbf{u}^{n+1}\right)\left(\mathbf{u}^{n+1}-\mathbf{u}^{n}\right) d x
\end{aligned}
$$

Upon exploiting (1.10) with $\mathbf{v}=\mathbf{u}^{n}$ and $\mathbf{u}=\mathbf{u}^{n+1}$, we arrive at energy dissipativity of the form

$$
\mathcal{E}\left(\mathbf{u}^{n+1}\right)-\mathcal{E}\left(\mathbf{u}^{n}\right) \leq 0
$$

Momentum conservation. Algorithm (2.18) preserves the linear momentum for $\gamma=2 \beta$. This is shown by testing the variational inclusion with 
constant $\mathbf{w} \in \mathbb{R}^{d}$, hence $\boldsymbol{\varepsilon}(\mathbf{w})=0$, so that

$$
\begin{aligned}
\mathbf{w} \cdot\left(\mathbf{L}^{n+1}-\mathbf{L}^{n}\right)= & \int_{\Omega}\left(\dot{\mathbf{u}}^{n+1}-\dot{\mathbf{u}}^{n}\right) \cdot \mathbf{w} d x \\
= & \int_{\Omega} \frac{2}{\tau}\left(\mathbf{u}^{n+1}-\mathbf{u}_{\mathrm{pred}}^{n+1}\right) \cdot \mathbf{w} d x \\
= & -\frac{1}{\tau}\left\{(1-2 \beta) \int_{\Omega} \boldsymbol{\sigma}\left(\mathbf{u}^{n}\right): \boldsymbol{\varepsilon}(\mathbf{w}) d x\right. \\
& +2 \beta \int_{\Omega} \boldsymbol{\sigma}\left(\mathbf{u}^{n+1}\right): \boldsymbol{\varepsilon}(\mathbf{w}) d x \\
& \left.-\int_{\Gamma_{C}^{S}} \boldsymbol{\nu}^{S} \cdot \boldsymbol{\sigma}\left(\mathbf{u}^{n+1, S}\right) \cdot \mathbf{w} d a-\int_{\Gamma_{C}^{M}} \boldsymbol{\nu}^{M} \cdot \boldsymbol{\sigma}\left(\mathbf{u}^{n+1, M}\right) \cdot \mathbf{w} d a\right\} \\
= & -\int_{\Gamma_{C}^{S}} \boldsymbol{\nu}^{S} \cdot \boldsymbol{\sigma}\left(\mathbf{u}^{n+1}\right) \cdot(\mathbf{w}-\mathbf{w}) d a=0
\end{aligned}
$$

From the discussion in Section 1.2 for the continuous case, the angular momentum cannot be expected to be preserved in general.

Artificial oscillations. There is clear numerical evidence that, depending on parameter specification, the above Algorithm (2.18) gives rise to unwanted oscillations in displacements and stresses (see also our numerical experiments in Section 3 below). This phenomenon, known as bouncing, can be extenuated by increasing $\gamma$ to values $\gamma>1 / 2$ at the expense of losing "second order consistency", which has anyway only been shown for the unconstrained case. In order to analyze this undesirable occurrence of oscillations assume that we have found contact over several timesteps so that

$$
\mathbf{u}^{n} \cdot \nu_{\phi}=\mathbf{u}^{n+1} \cdot \nu_{\phi} .
$$

Then, the update formula for the velocities, third line of Algorithm (2.18), yields

$$
\begin{aligned}
\dot{\mathbf{u}}^{n+1} \cdot \nu_{\phi} & =\dot{\mathbf{u}}^{n} \cdot \nu_{\phi}-\tau\left(\mathbf{F}^{1 / 2}\left(\mathbf{u}^{n}, \mathbf{u}^{n+1}\right)-\mathbf{F}_{\mathrm{con}}\left(\mathbf{u}^{n+1}\right)\right) \cdot \nu_{\phi} \\
& =\dot{\mathbf{u}}^{n} \cdot \nu_{\phi}-\tau\left[\frac{2}{\tau^{2}}\left(\mathbf{u}^{n+1}-\left(\mathbf{u}^{n}+\tau \dot{\mathbf{u}}^{n}\right)\right)\right] \cdot \nu_{\phi},
\end{aligned}
$$

which directly leads to

$$
\dot{\mathbf{u}}^{n+1} \cdot \nu_{\phi}=-\dot{\mathbf{u}}^{n} \cdot \nu_{\phi} .
$$

This is a zigzagging of the normal component of the velocities as observed in numerical experiments, see Figure 4, left, in Section 3 below. 


\subsection{Contact stabilization}

In the following we assume that the spatial quantities as $\mathbf{u}^{n}$ are obtained after spatial discretization by finite elements $\mathbf{S}_{h}, h>0$ the discretization parameter. Let us note that also $\mathcal{K} \subset \mathbf{S}_{h}$ has to be understood as a discrete approximation of the set of admissible displacements (1.5). For details concerning the spatial discretization, we refer the reader to $[8,10]$

The insight obtained by the analysis at the end of Section 2.2 directly guides us to a further modification of the Newmark scheme. This modification is inspired by the physically reasonable requirement that the normal components of the forces acting at the contact boundary should balance, i.e.

$$
\mathbf{F}^{1 / 2}\left(\mathbf{u}^{n}, \mathbf{u}^{n+1}\right) \cdot \nu_{\phi}=\mathbf{F}_{\mathrm{con}}\left(\mathbf{u}^{n+1}\right) \cdot \nu_{\phi} .
$$

This motivates us to replace the linear predictor step in the first line of Algorithm (2.18) by a nonlinear one, which is realized by means of an $L^{2}$ projection in $\mathbf{S}_{h}$. This modification gives rise to our

\section{Contact-stabilized Newmark algorithm:}

$$
\begin{aligned}
0 & \in \mathbf{u}_{\text {pred }}^{n+1}-\left(\mathbf{u}^{n}+\tau \dot{\mathbf{u}}^{n}\right)+\partial I_{\mathcal{K}}\left(\mathbf{u}_{\text {pred }}^{n+1}\right) \\
0 & \in \mathbf{u}^{n+1}-\mathbf{u}_{\text {pred }}^{n+1}+\frac{1}{2} \tau^{2}\left(\mathbf{F}^{1 / 2}\left(\mathbf{u}^{n}, \mathbf{u}^{n+1}\right)+\partial I_{\mathcal{K}}\left(\mathbf{u}^{n+1}\right)\right) \\
\dot{\mathbf{u}}^{n+1} & =\dot{\mathbf{u}}^{n}-\tau\left(\mathbf{F}^{1 / 2}\left(\mathbf{u}^{n}, \mathbf{u}^{n+1}\right)-\mathbf{F}_{\text {con }}\left(\mathbf{u}^{n+1}\right)\right) .
\end{aligned}
$$

Remark. The variational inclusion defining the modified predictor in Algorithm (2.21) requires the evaluation of the normal trace of $\mathbf{u}$. Although the trace of a finite element function in $\mathbf{S}_{h}$ is always well-defined, this is not the case for an arbitrary $L^{2}$-function. This corresponds to the fact that the values of a function $\mathbf{u} \in \mathbf{S}_{h}$ at the boundary also serve as values of the finite element function within the small strip given by the elements at the boundary. It is this double role of the degrees of freedom on the boundary as interior and boundary values, which, on one hand, causes artificial oscillations at the contact interface and, on the other hand, allows for removing the oscillations by means of the discrete $L^{2}$-projection given above.

Again, in the spirit of our definition of $\mathbf{F}_{\text {con }}(\mathbf{u})$, once the variational inclusion in the first line of (2.21) has been solved, we may characterize its solution in terms of some $\mathbf{G}_{\text {con }}$ such that

$$
\left(\mathbf{G}_{\mathrm{con}}\left(\mathbf{u}_{\text {pred }}^{n+1}\right), \mathbf{v}\right)_{\mathbf{L}^{2}}=\left(\mathbf{u}^{n}+\tau \dot{\mathbf{u}}^{n}-\mathbf{u}_{\text {pred }}^{n+1}, \mathbf{v}\right)_{\mathbf{L}^{2}}
$$


Upon rewriting the variational inclusion in the first line of (2.21) as variational inequality we get

$$
\left(\mathbf{u}_{\text {pred }}^{n+1}, \mathbf{v}-\mathbf{u}_{\text {pred }}^{n+1}\right)_{\mathbf{L}^{2}} \geq\left(\mathbf{u}^{n}+\tau \dot{\mathbf{u}}^{n}, \mathbf{v}-\mathbf{u}_{\text {pred }}^{n+1}\right)_{\mathbf{L}^{2}}, \quad \forall \mathbf{v} \in \mathcal{K}
$$

which directly leads to

$$
\left(\mathbf{G}_{\text {con }}\left(\mathbf{u}_{\text {pred }}^{n+1}\right), \mathbf{v}-\mathbf{u}_{\text {pred }}^{n+1}\right)_{\mathbf{L}^{2}} \leq 0, \quad \forall \mathbf{v} \in \mathcal{K} .
$$

Theorem 2.2 Consider Algorithm (2.21). If no contact occurs, this algorithm is energy conserving. If contact occurs, it can be energy dissipative.

Proof 2.2 Following similar lines as in the proof of the above Theorem 2.1, we here obtain

$$
\begin{aligned}
\dot{\mathbf{u}}^{n+1}-\dot{\mathbf{u}}^{n} & =\frac{2}{\tau}\left(\mathbf{u}^{n+1}-\mathbf{u}_{\text {pred }}^{n+1}\right) \\
\dot{\mathbf{u}}^{n+1}+\dot{\mathbf{u}}^{n} & =\frac{2}{\tau}\left(\mathbf{u}^{n+1}-\mathbf{u}^{n}+\mathbf{G}_{\mathrm{con}}\left(\mathbf{u}_{\text {pred }}^{n+1}\right)\right) .
\end{aligned}
$$

As before

$$
\begin{aligned}
\mathcal{E}_{\text {kin }}\left(\mathbf{u}^{n+1}\right)-\mathcal{E}_{\text {kin }}\left(\mathbf{u}^{n}\right)= & -\frac{1}{2} a\left(\mathbf{u}^{n+1}+\mathbf{u}^{n}, \mathbf{u}^{n+1}-\mathbf{u}^{n}\right) \\
& +f_{\text {ext }}\left(\mathbf{u}^{n+1}-\mathbf{u}^{n}\right)+\int_{\Omega} \mathbf{F}_{\text {con }}\left(\mathbf{u}^{n+1}\right)\left(\mathbf{u}^{n+1}-\mathbf{u}^{n}\right) d x \\
& +\frac{2}{\tau^{2}} \int_{\Omega} \mathbf{G}_{\text {con }}\left(\mathbf{u}_{\text {pred }}^{n+1}\right)\left(\mathbf{u}^{n+1}-\mathbf{u}_{\text {pred }}^{n+1}\right) d x .
\end{aligned}
$$

Finally, we end up with

$$
\begin{aligned}
\mathcal{E}\left(\mathbf{u}^{n+1}\right)-\mathcal{E}\left(\mathbf{u}^{n}\right)= & \int_{\Omega} \mathbf{F}_{\text {con }}\left(\mathbf{u}^{n+1}\right)\left(\mathbf{u}^{n+1}-\mathbf{u}^{n}\right) d x \\
& +\frac{2}{\tau^{2}} \int_{\Omega} \mathbf{G}_{\text {con }}\left(\mathbf{u}_{\text {pred }}^{n+1}\right)\left(\mathbf{u}^{n+1}-\mathbf{u}_{\text {pred }}^{n+1}\right) d x \\
\leq & 0 .
\end{aligned}
$$

Here, in the last line we have exploited (2.22).

Note that even in the occurrence of contact the energy is conserved, when the contact boundary predicted by $\mathbf{u}_{\text {pred }}^{n+1}$ coincides with the one given by $\mathbf{u}^{n+1}$. 
Avoidance of artificial oscillations. In contrast to (2.19), which gives rise to artificial oscillations in Algorithm (2.18), the force equilibrium (2.20) here immediately leads to the desirable property

$$
\dot{\mathbf{u}}^{n+1} \cdot \nu_{\phi}=\dot{\mathbf{u}}^{n} \cdot \nu_{\phi}
$$

Remark. If the above global condition (2.20) is realized in a time discretization with lumped mass matrix, then it may be interpreted as pointwise local projection onto the set of admissible displacements. We want to explicitly mention that this feature nicely agrees with suggestions made by $[6]$ (see Figure 3.8 therein). In this case, the cost for computing the modified predictor in Algorithm (2.21) is negligible, since all unknowns decouple and the nonlinear projection can be carried out pointwise on the boundary.

\section{A two body Hertzian contact problem}

In this section, we compare two recently suggested modifications to the classical Newmark method:

old the dissipative Algorithm (2.18) due to Kane et al. [6],

new the dissipative and contact-stabilized Algorithm (2.21), as suggested here.

The implementation of our algorithm has been done both within the framework of the finite element toolbox UG [1] and the obstacle toolbox OBSLIB, see [11]. Let us mention that our implementation of the algorithms given in Section 2 is not restricted to the two dimensional case. As an example, we refer to [9], where the stabilized Algorithm (2.21) has been used for the three dimensional simulation of a contact problem in biomechanics.

As an illustrative test problem we selected a two body Hertzian contact problem in $2 D$ with the following specifications: At initial time $t=0$, two circles $\Omega^{S}, \Omega^{M}$ with radii $r=8$, midpoints on the $y$-axis, and initial distance 1.5 are moving with vertical speed $v_{0, S}=-1, v_{0, M}=+1$, respectively. The computational meshes are shown in Figure 2. The possible contact boundaries are the lower and upper quarters of the boundaries of $\Omega_{S}$ and $\Omega_{M}$, respectively. On the remaining parts of the boundaries, homogeneous Neumann boundary conditions are prescribed and we set the volume forces to 
$\mathbf{f}=0$. As material parameters we choose $E=5 \cdot 10^{2}$ and $\nu=0.3$ on both domains. The computations are carried out until $T=5$. For the discretiza-

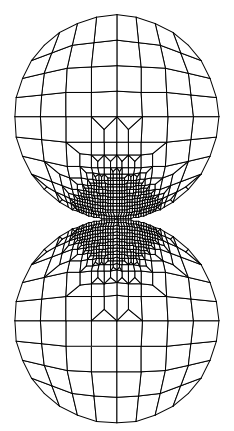

Figure 2: Computational meshes of the two body Hertzian contact problem.

tion in time, we choose the constant step size $\tau=5 \cdot 10^{-4}$ and consider the dissipative modification (2.18) as well as our stabilized scheme (2.21). The discretization in space is done by bilinear finite elements on quadrilaterals, which in general behave less stiff than triangles. The information transfer at the contact interface $\Gamma_{C}$ is realized by means of nonconforming domain decomposition or mortar methods, see [15]. Among the possible solvers for variational inequalities, we selected monotone multigrid methods [10], which have the nice feature that they do not require any regularization parameters to be chosen. These methods allow for the solution of nonlinear contact problems with multigrid efficiency, thus leading to an overall highly efficient method. For the $L^{2}$-scalar product, we use a lumped mass matrix, which makes the cost for computing Algorithm (2.21) instead of Algorithm (2.18) negligible, see the remark at the end of Section 2.

Energy dissipation. In Figure 3, the total energy for the dissipative Newmark scheme (2.18) and the dissipative contact-stabilized scheme (2.21) are shown. Compared to the older version (2.18), our new version (2.21) generates a slightly smaller loss of energy during the initial contact phase but a higher loss when the two discs separate. Following the analysis in Section 2, the contact-stabilized scheme (2.21) might be expected to lose more energy than the scheme (2.18) in each time step. However, this does not mean that the total energy arising from the stabilized scheme (2.21) always has to be smaller than the total energy created by (2.18), since both methods yield 


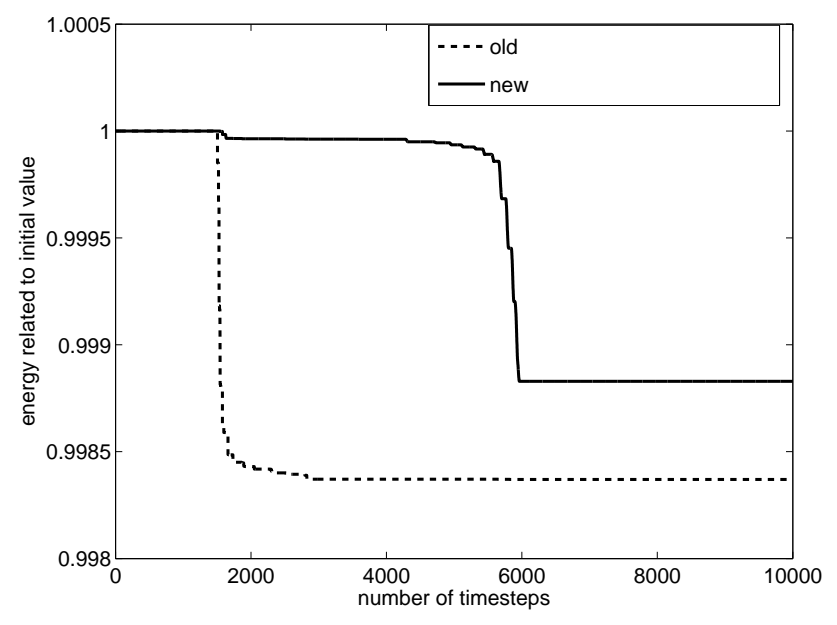

Figure 3: Comparative behavior of total energy

in general different trajectories. In any case, both schemes yield reasonable results and generate comparable energy losses of less than $0.2 \%$.

Artificial oscillations. Figure 4 shows the number of contact points, i.e., the number of vertices $\Gamma_{C}^{S}$ where contact actually occurs, over a typical segment of time steps. As can be observed, the old Algorithm suffers from artificial oscillations caused by the undesirable property (2.19), whereas the new algorithm only shows very few oscillations, in agreement with desirable property $(2.20)$.
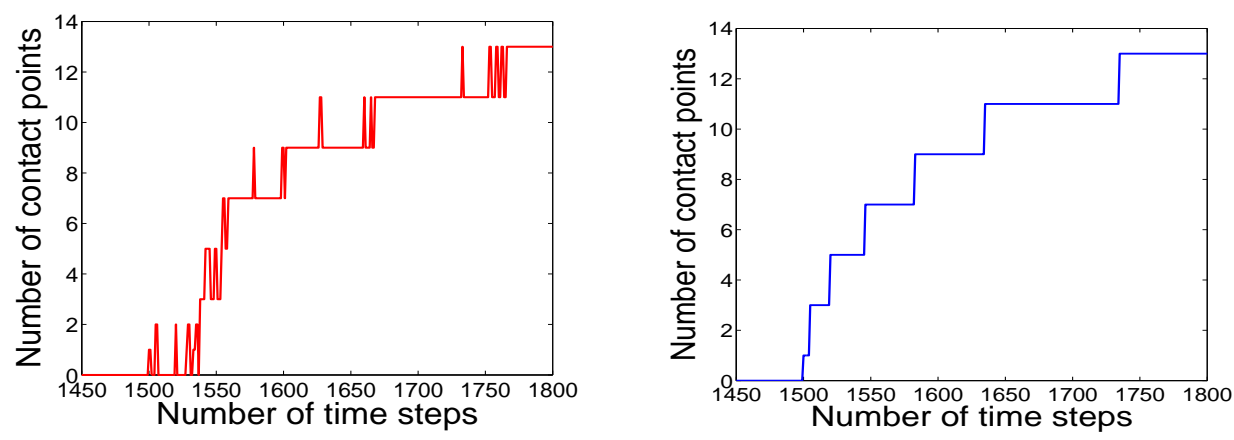

Figure 4: Number of contact points. Left: old Algorithm (2.18): 400 oscillations. Right: new Algorithm (2.21): 3 oscillations. 
This effect is also confirmed by a reduction of oscillations in the tangential displacements, see Figure 5, where, the tangential displacements of the point at the south pole of $\Omega^{M}$ are depicted. Once again, the spurious oscillations generated by the Newmark scheme (2.18) are nearly completely removed by the contact-stabilized Algorithm (2.21).
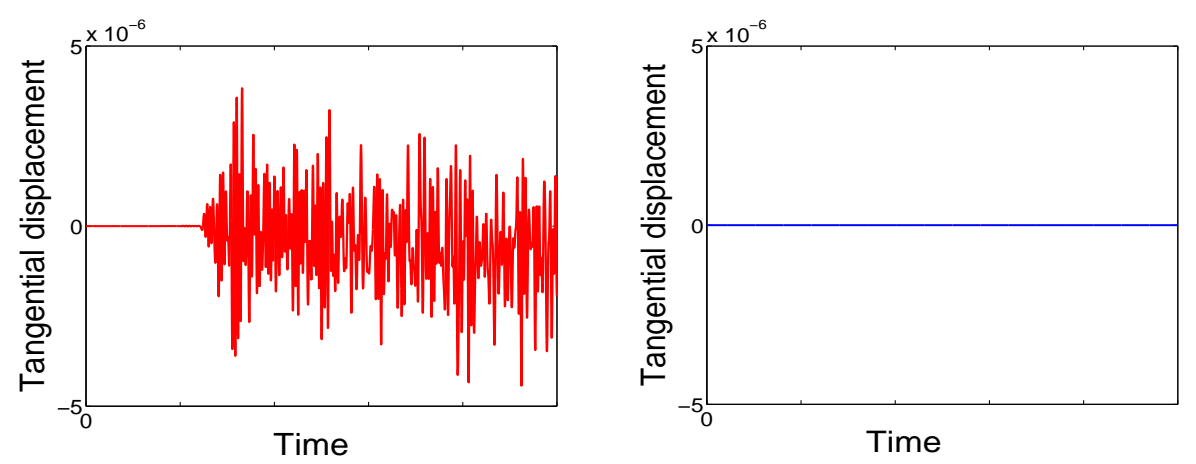

Figure 5: Tangential displacements at the south pole of $\Omega^{S}$. Left: old Algorithm (2.18). Right: new Algorithm (2.21).

Boundary stresses and total contact force. In Figure 6, we compare the total contact force $\int_{\Gamma_{C}^{S}} \sigma_{n} d a$ generated by the old scheme $(2.18)$ and by our new scheme (2.21). At the time when the two bodies come into contact, extremely high values of the total contact force can be observed for the old scheme. This overshooting phenomenon is clearly eliminated by the new scheme. The remaining oscillations in the total contact forces might originate from vibrations within the elastic material. Even more insight is gained from the zoom in Figure 7, where the zigzagging from the old algorithm is contrasted with the smooth behavior of the new one. Clearly, the oscillations arising from the old algorithms are revealed to be artificial.

Acknowledgments One of us (R.K.) gratefully acknowledges support by a Konrad Zuse Fellowship. We want to thank Ralf Kornhuber for many fruitful discussions on the topic of this paper and Mirjam Walloth for careful reading. 

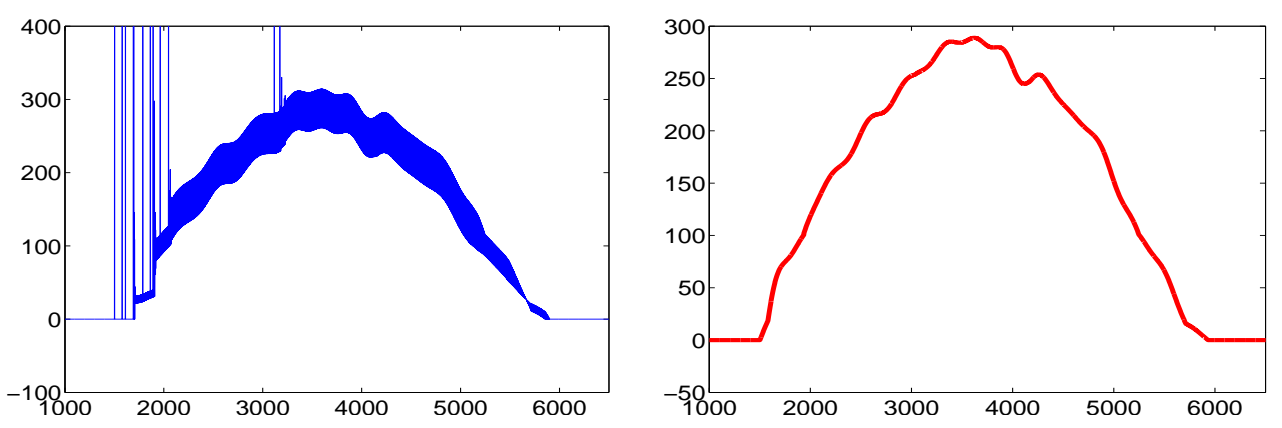

Figure 6: Comparison of total contact forces. Left: old Algorithm (2.18). Right: new Algorithm (2.21).

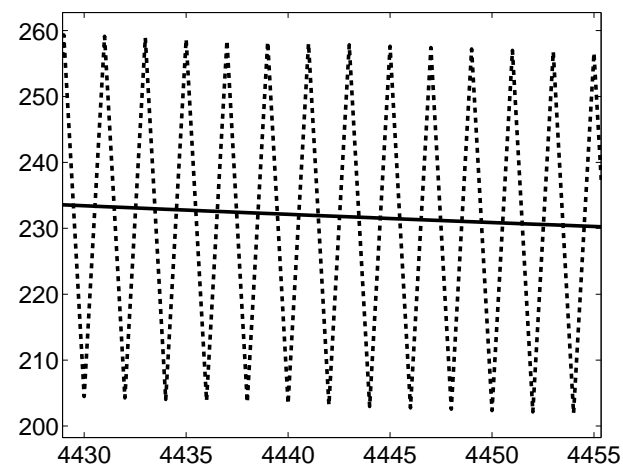

Figure 7: Comparison of total contact forces (zoom, overlay of Figure 6 , left and right): old (- - ) versus new algorithm (-)

\section{References}

[1] P. Bastian, K. Birken, K. Johannsen, S. Lang, N. Neuß, H. RentzReichert, and C. Wieners. UG - a flexible software toolbox for solving partial differential equations. Computing and Visualization in Science, 1:27-40, 1997.

[2] G. Duvaut and J.L. Lions. Inequalities in Mechanics and Physics. Springer, 1976.

[3] Christof Eck. Existenz und Regularität der Lösungen für Kontaktprobleme mit Reibung. PhD thesis, Universität Stuttgart, 1996. 
[4] I. Ekeland and R. Temam. Convex Analysis and Variational Problems. North-Holland, Amsterdam, 1976.

[5] E. Hairer, C. Lubich, and G. Wanner. Geometric Numerical Integration. Structure-Preserving Algorithms for Ordinary Differential Equations. Computational Mathematics. Springer, Berlin, Heidelberg, 2nd edition, 2006.

[6] C. Kane, E.A. Repetto, M. Ortiz, and J.E. Marsden. Finite element analysis of nonsmooth contact. Comp. Meth. Appl. Mech. Eng., 180:126, 1999.

[7] H. B. Khenous, P. Laborde, and Y. Renard. On the discretization of contact problems in elastodynamics. Technical report, INSA, Toulouse, 2006 .

[8] N. Kikuchi and J.T. Oden. Contact Problems in elasticity. SIAM, Philadelphia, 1988.

[9] R. Kornhuber, R. Krause, P. Deuflhard, O. Sander, and S. Ertel. A Monotone Multigrid Solver for Two Body Contact Problems in Biomechanics. Comp. Vis. Sci., 2006.

[10] Ralf Kornhuber and Rolf Krause. Adaptive multilevel methods for Signorini's problem in linear elasticity. Comp. Vis. Sci., 4:9-20, 2001.

[11] R. Krause. Monotone Multigrid Methods for Signorini's Problem with Friction. $\mathrm{PhD}$ thesis, FU Berlin, 2000. http://www.diss.fuberlin.de/2001/240/indexe.html.

[12] T. A. Laursen. Computational Contact and Impact Mechanics. SpringerVerlag, Berlin Heidelberg New York, 2003.

[13] T. A. Laursen and V. Chawla. Design of energy conserving algorithms for frictionless dynamic contact problems. International Journal for Numerical Methods in Engineering, 40:863-886, 1997.

[14] J. Marsden and M. West. Discrete mechanics and variational integrators. Acta Num., 10:357-514, 2001.

[15] B. Wohlmuth and R. Krause. Monotone methods on nonmatching grids for nonlinear contact problems. SISC, 25(1):324-347, 2003. 